\title{
Welcome message from the Editor-in-Chief
}

\author{
Jun Zheng
}

National Mobile Communications Research Lab, School of Information Science and Engineering, Southeast University, 2 Si Pai Lou, Nanjing, Jiangsu 210096, China

Received on 3 October 2011

Copyright (c) 2011 Zheng, licensed to ICST. This is an open access article distributed under the terms of the Creative Commons Attribution licence (http://creativecommons.org/licenses/by/3.0/), which permits unlimited use, distribution and reproduction in any medium so long as the original work is properly cited.

doi: 10.4108/icst.trans.mca.2011.el

First of all, congratulations to the launch of ICST Transactions on Mobile Communications and Applications, and welcome to the inaugural issue of this new research journal!

With significant advances in wireless communication and networking technologies, the last two decades have witnessed a rapid growth of mobile communications and its applications. The emergence and development of a variety of wireless communication systems and networks have enabled many envisioned mobile applications, which have fundamentally changed and significantly improved the way people work and live. For example, cellular communication systems have experienced three generations from $1 \mathrm{G}, 2 \mathrm{G}$ to $3 \mathrm{G}$ and are now evolving toward the $4 \mathrm{G}$ era, aiming to provide higher-rate and better-quality communication service to subscribers. Satellite communication systems, which have large coverage, have been widely used in a variety of civilian, commercial, and military areas, such as television broadcast, satellite positioning, and military communication. Mobile ad hoc networks, which do not rely on any existing network infrastructure and thus are convenient for deployment, promise to enable a broad range of communication applications in special situations, such as battle fields and disaster regions. Despite the significant advances, however, the ever-increasing demands for a broader range of applications and better services have presented a plethora of new challenges for mobile communication technologies, which have motivated tremendous research work in our community.

ICST Transactions on Mobile Communications and Applications is a new open access scholarly archival journal that is committed to the timely publication of high-quality original research papers on all aspects of mobile communications theories, technologies, systems, and applications. Original contributions that have not been published and are not currently under consideration by any other journal are solicited. All submissions will go through a rigorous peer-review process, and will be reviewed and evaluated

Email: junzheng@seu.edu.cn by expert referees and the editorial board. Areas of interests include, but are not limited to:

- Mobile cellular networks

- Mobile ad hoc networks

- Mobile Internet

- WiMAX networks

- Vehicular networks

- Femtocell networks

- Wireless sensor networks

- Cognitive radio networks

- Satellite communications systems

- Mobile system and network architectures

- Network control and management

- Spectrum and resource management

- Mobility management and localization

- Modulation and coding techniques

- Quality of service

- Reliability and fault tolerance

- Security and privacy

- Mobile applications and services

- Performance modeling and analysis

- Wireless network measurement and inference

- Testbed, prototyping, and performance benchmark

This inaugural issue includes a collection of five outstanding papers that are contributed by leading researchers and cover a diversity of topics in the area of wireless networks. I hope that you will find the papers timely, informative, and enjoyable. 
I would like to take this opportunity to acknowledge all the authors who contributed to this inaugural issue of the journal. I am grateful to all anonymous reviewers for their time and effort in reviewing the papers and providing us and the authors valuable review comments. I am thankful to all editorial board members for their strong support in founding this new journal. I am also thankful to all ICST publication staff, in particular, Dr. Joseph Wakeling, for their great efforts and assistance in producing and launching this inaugural issue of the journal.

Finally, I cordially welcome all readers and fellow researchers to submit your papers and contribute your work to this journal. I am also keen to hear your constructive ideas and suggestions for helping the growth of this new born but promising research journal.

Thank you all. I look forward to your contributions.

\section{About the Editor-in-Chief}

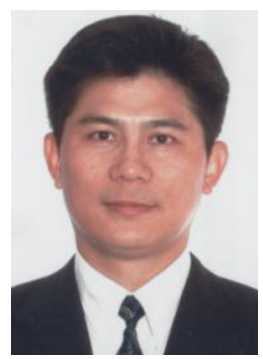

Jun Zheng is a full professor with the National Mobile Communications Research Laboratory, Southeast University (SEU), Nanjing, China. $\mathrm{He}$ received his Ph.D. degree in electrical and electronic engineering from The University of Hong Kong, Hong Kong, China. Before joining SEU, he was with the School of Information Technology and Engineering, University of Ottawa, Ottawa, Canada.

He has conducted extensive research in the field of communications and communication networks. The scope of his research includes design and analysis of network architectures and protocols for efficient and reliable communications and their application to different types of networks. He has co-authored the book Wireless Sensor Networks: A Networking Perspective (Wiley-IEEE Press, 2009) and the book Optical WDM Networks: Concepts and Design Principles (Wiley-IEEE Press, 2004), and has published over 100 technical papers in refereed journals and magazines, and peer-reviewed conference proceedings. His current research interests include mobile communication networks, wireless sensor networks, and mobile ad hoc networks, focused on network architectures and protocols.

He serves as a Technical Editor of IEEE Communications Magazine and an editorial board member of several other refereed journals, including Springer Wireless Networks and Applications, Elsevier Ad Hoc Networks Journal, and Wiley Wireless Communications and Mobile Computing. He was an Editor of IEEE Communications Surveys \& Tutorials and an Associate Editor of IEEE/ OSA Journal of Optical Communications and Networking. He has co-edited 10 special issues for different refereed journals and magazines, including a feature topic issue of IEEE Communications Magazine on the Internet of Things, a special issue of IEEE Network on Wireless Sensor Networking, a special issue of IEEE Network on Advances in Broadband Access Networks, a special issue of IEEE JSAC on Network Coding for Wireless Communication Networks, and a special issue of IEEE JSAC on Broadband Access Networks: Architectures and Protocols, all as Lead Guest Editor. He was the founding General Chair of AdHocNets'09 and General Chair of AccessNets'07, and has served as TPC Co-Chair and Symposium Co-Chair of many international conferences and symposia, including IEEE GLOBECOM'08, '10, and '12, and ICC'09 and ' 11 . He has also served on the technical program committees of a number of international conferences and symposia. He is a Senior Member of the IEEE and IEEE Communications Society. 